\title{
On the Stability and Accuracy of One-Step Methods for Solving Stiff Systems of Ordinary Differential Equations
}

\author{
By A. Prothero and A. Robinson
}

\begin{abstract}
The stiffness in some systems of nonlinear differential equations is shown to be characterized by single stiff equations of the form

$$
y^{\prime}=g^{\prime}(x)+\lambda\{y-g(x)\} .
$$

The stability and accuracy of numerical approximations to the solution $y=g(x)$, obtained using implicit one-step integration methods, are studied. An $S$-stability property is introduced for this problem, generalizing the concept of $A$-stability. A set of stiffly accurate onestep methods is identified and the concept of stiff order is defined in the limit $\operatorname{Re}(-\lambda) \rightarrow \infty$. These additional properties are enumerated for several classes of $A$-stable one-step methods, and are used to predict the behaviour of numerical solutions to stiff nonlinear initial-value problems obtained using such methods. A family of methods based on a compromise between accuracy and stability considerations is recommended for use on practical problems.
\end{abstract}

1. Introduction. The study of numerical methods for integrating stiff systems of ordinary differential equations has centred largely on the concept of $A$-stability proposed by Dahlquist [1]. As Dahlquist also showed that the maximum order of an $A$-stable linear multistep method is two, subsequent research on higher-order methods has concentrated either on the formulation of multistep methods satisfying some less restrictive stability condition (e.g., Widlund [2], Norsett [3], Gear [4]), or on the study of other classes of integration methods which combine $A$-stability with high-order accuracy (e.g., Treanor [5], Norsett [6], Ehle [7], [8], Axelsson [9], [10], Chipman [11], [12] and Watts and Shampine [13]). Much of the work in this latter category has concerned the $A$-stability properties of implicit one-step methods ([7]-[13]); several classes of such methods, containing processes of arbitrarily high order, have been shown to be $A$-stable.

In using $A$-stable one-step methods to solve large systems of stiff nonlinear differential equations [14], we have found that

(a) some $A$-stable methods give highly unstable solutions, and

(b) the accuracy of the solutions obtained when the equations are stiff often appears to be unrelated to the order of the method used.

This has caused us to re-examine the form of stability required when stiff systems of equations are solved, and to question the relevance of the concept of (nonstiff) order of accuracy for stiff problems.

Received February 9, 1973.

AMS (MOS) subject classifications (1970). Primary 65L05; Secondary 34A50, 34D05, 34E05.

Key words and phrases. Stiff system of ordinary differential equations, implicit one-step methods, $A$-stability, $S$-stability, stiffly accurate methods, stiff order.

Copyright $\odot$ 1974, American Mathematical Society 
We consider an initial-value problem involving a set of ordinary differential equations

$$
\mathbf{y}^{\prime}=\mathrm{f}(x, \mathrm{y}), \quad \mathrm{y}=\mathrm{y}_{0} \quad \text { at } x=0 .
$$

This set of equations may be approximated, in the neighbourhood of the solution $\mathrm{y}=\mathbf{g}(x)$, by

$$
\begin{aligned}
\mathbf{y}^{\prime} & \approx \mathbf{f}(x, \mathrm{~g}(x))+J(x)\{\mathrm{y}-\mathrm{g}(x)\} \\
& =\mathbf{g}^{\prime}(x)+J(x)\{\mathbf{y}-\mathbf{g}(x)\},
\end{aligned}
$$

where $J$ denotes the variational (or Jacobian) matrix

$$
J(x)=\mathrm{f}_{\mathrm{y}}(x, \mathrm{~g}(x)) .
$$

The problem (1.1) is termed "stiff" if the eigenvalues $\lambda(x)$ of the variational matrix $J(x)$ are such that

$$
\underset{\lambda}{\operatorname{Max}}\{\operatorname{Re}(-\lambda(x))\} \gg \underset{\lambda}{\operatorname{Max}}\{\operatorname{Re}(\lambda(x))\}
$$

over some range of $x$ in the required range of the solution. Now, in many problems in which the eigenvalues are widely spread in the sense (1.4), it is possible to define a subset $S$ of "stiff eigenvalues" such that

$$
-\underset{\lambda \in S}{\operatorname{Re}}\{\lambda(x)\} \gg \operatorname{Max}_{\lambda \notin S}|\operatorname{Re}\{\lambda(x)\}|=\bar{\lambda}(x),
$$

i.e., the stiff eigenvalues $S$ are "widely separated" from the remainder. In such a situation, there exists a matrix $J_{s}(x)$, with nonzero eigenvalues equal to the eigenvalues $\lambda(x) \in S$, such that

$$
J_{s}(x)=J(x)+O(\bar{\lambda}(x)) .
$$

The greater the separation between the two sets of eigenvalues in (1.5), the nearer are Eqs. (1.2) in form to the set of equations

$$
\mathbf{y}^{\prime}=\mathbf{g}^{\prime}(x)+J_{s}(x)\{\mathbf{y}-\mathbf{g}(x)\},
$$

and the more closely may we expect the Eqs. (1.7) to characterize the stiffness properties of the system of nonlinear Eqs. (1.1). The numerical difficulties arising from the stiffness of Eqs. (1.1) may thus be directly related to the problems of solving the set of Eqs. (1.7), and these may be analysed effectively in terms of the stability and accuracy of numerical solutions to a single equation of the form

$$
y^{\prime}=g^{\prime}(x)+\lambda(x)\{y-g(x)\},
$$

obtained using an integration step-size $h$ such that $h \operatorname{Re}(-\lambda) \gg 1$.

To a first approximation, we may neglect the dependence of $\lambda$ on $x$ in Eq. (1.8). The rate of change of $\lambda$ with $x$ is in general of the same order of magnitude as $g^{\prime}(x)$, and the integration step-size is such that $h g^{\prime}(x) \ll 1$, so that

$$
|d \lambda / d x| \ll \operatorname{Re}(-\lambda) .
$$

In this paper, therefore, we examine the stability and accuracy of numerical approximations, using implicit one-step methods, to the solution $y=g(x)$ of the dif- 
ferential equation

$$
y^{\prime}=g^{\prime}(x)+\lambda\{y-g(x)\},
$$

where $g^{\prime}(x)$ is any given bounded function, for complex $\lambda$ such that $h \operatorname{Re}(-\lambda) \gg 1$.

We show that the $A$-stability property [1], while necessary, does not ensure the stability of solutions to Eq. (1.9) for $g^{\prime}(x) \not \equiv 0$, and we derive necessary and sufficient conditions for such stability (which we term $S$-stability). Conditions for strong (or stiff) $S$-stability are also proved.

The accuracy of numerical solutions to Eq. (1.9) is treated by considering the asymptotic form of the local truncation error, proportional to $h^{s+1} \lambda^{t}$, in the limit $h \operatorname{Re}(-\lambda) \rightarrow \infty$ and $h \rightarrow 0$. A subset of stiffly accurate one-step methods is defined for which $t=-1$, and maximum values on the effective stiff order $s$ are obtained.

We then determine the $S$-stability and stiff order properties of several classes of implicit one-step methods, based on Gauss-Legendre, Radau and Lobatto quadrature formulae. All the classes studied have been shown to be $A$-stable ([7], [9], [10]), but significant differences in their $S$-stability and stiff-accuracy properties give us good reason to prefer two particular classes for solving stiff equations, both of the linearized form (1.9) and, for the reasons developed above, of the general nonlinear form (1.1).

2. Stability of One-Step Methods. An $r$-stage one-step method for the numerical solution of a first-order differential equation

$$
y^{\prime}=f(x, y)
$$

may be expressed in the general form

$$
y_{n+1}=y_{n}+\sum_{i=1}^{r} b_{i} k_{i}
$$

where

$$
k_{i}=h f\left(x_{n}+h c_{i}, y_{n}+\sum_{i=1}^{r} a_{i j} k_{i}\right) \quad(i=1,2, \cdots, r)
$$

and $h=x_{n+1}-x_{n}$. The $r \times r$ array $A=\left(a_{i j}\right)$ and the vectors $\mathbf{b}^{T}=\left(b_{1}, b_{2}, \cdots, b_{r}\right)$ and $\mathbf{c}^{T}=\left(c_{1}, c_{2}, \cdots, c_{r}\right)$ are constants satisfying $c_{i}=\sum_{j=1}^{r} a_{i j}$, the values of which uniquely define a particular one-step method.

We wish to categorize those one-step methods which give a stable numerical solution when applied with positive step-size to any equation of the form

$$
y^{\prime}=g^{\prime}(x)+\lambda\{y-g(x)\},
$$

where $\lambda$ is a complex constant with negative real part, and where $g^{\prime}(x)$ is any function that is defined and bounded in some interval $x \in[0, \bar{x}]$. To this end, it is necessary to define a stability property, termed $S$-stability, which generalizes to equations of the form (2.4) the concept of $A$-stability introduced by Dahlquist [1] for the related equations $y^{\prime}=\lambda y$.

Definitions. A one-step method (2.2) is said to be S-stable if, for a differential equation of the form (2.4) and for any real positive constant $\lambda_{0}$, there exists a real positive constant $h_{0}$ such that 


$$
\| \frac{y_{n+1}-g\left(x_{n+1}\right)}{y_{n}-g\left(x_{n}\right)}||<1,
$$

provided $y_{n} \neq g\left(x_{n}\right)$, for all $0<h<h_{0}$ and all complex $\lambda$ with $\operatorname{Re}(-\lambda) \geqq \lambda_{0}, x_{n}$, $x_{n+1} \in[0, \bar{x}]$. If the above conditions only hold for $|\arg (-h \lambda)|<\alpha$, then the method is said to be $S(\alpha)$-stable.

An $S$-stable one-step method is said to be strongly $S$-stable if

$$
\frac{y_{n+1}-g\left(x_{n+1}\right)}{y_{n}-g\left(x_{n}\right)} \rightarrow 0
$$

as $\operatorname{Re}(-\lambda) \rightarrow \infty$, for all positive $h$ such that $x_{n}, x_{n+1} \in[0, \bar{x}]$.

It may be seen that, for $g^{\prime}(x) \equiv 0$, these definitions are equivalent to the definitions of $A$-stability and strong $A$-stability for one-step methods.

When the one-step method (2.2) is applied to Eq. (2.4), the vector $\mathbf{k}^{T}=$ $\left(k_{1}, \cdots, k_{r}\right)$ is given by

$$
(I-h \lambda A) \mathbf{k}=h \mathbf{g}^{\prime}+h \lambda\left\{y_{n} \mathbf{e}-\mathbf{g}\right\}
$$

where $\mathbf{g}^{T}=\left(g\left(x_{1}\right), \cdots, g\left(x_{r}\right)\right)$ with $x_{i}=x_{n}+h c_{i}, \mathbf{g}^{\prime}$ is similarly defined, and $\mathrm{e}^{T}=(1, \cdots, 1)$. Equation $(2.5)$ defines $\mathbf{k}$ uniquely for positive $h$ and for all $\lambda$ with $\operatorname{Re}(\lambda)<0$ only if the array $A$ has nonnegative eigenvalues. A one-step method with positive semidefinite or positive definite array $A$ is therefore termed "welldefined" and, for such a method, the increment

$$
\mathbf{b}^{T} \mathbf{k}=\mathbf{b}^{T}(z I-A)^{-1}\left\{\epsilon_{n} \mathbf{e}+g\left(x_{n}\right) \mathbf{e}-\mathbf{g}+h z \mathbf{g}^{\prime}\right\},
$$

where $z=(h \lambda)^{-1}$ and $\epsilon_{n}=y_{n}-g\left(x_{n}\right)$, is uniquely defined for all complex $z$ with $\operatorname{Re}(z)<0$.

For simplicity, we assume that the abscissae $c_{i}$ of the one-step methods lie in the interval $[0,1]$, although this restriction need not be made provided $g(x)$ and $g^{\prime}(x)$ are defined and bounded over an appropriately wider range of $x$. Also, without loss of generality, we order the abscissae so that $c_{i} \leqq c_{i}$ if $i<j$. Let there be $r^{*}$ $\left(1 \leqq r^{*} \leqq r\right)$ different abscissae $\mathrm{c}^{*}$ in $\mathrm{c}$, and define an $r \times r^{*}$ array $E(z)$ by

$$
\begin{aligned}
E_{i i} & =-z & & \text { for } c_{i}=c_{i}^{*}=0, \\
& =c_{i} & & \text { for } c_{i}=c_{i}^{*} \neq 0, \\
& =0 & & \text { otherwise. }
\end{aligned}
$$

Using Eq. (2.6), the difference equation (2.2) may be expressed in the form

$$
\epsilon_{n+1}=\left(1-\mathbf{b}^{T}(A-z I)^{-1} \mathbf{e}\right) \epsilon_{n}-h G_{0}+h \mathrm{~b}^{T}(A-z I)^{-1} E(z)\left\{\mathbf{G}_{1}-z \mathrm{G}_{2}\right\},
$$

with the dependence on the function $g(x)$ contained in

$$
h G_{0}=g\left(x_{n}+h\right)-g\left(x_{n}\right)
$$

and the $r^{*}$-vectors $G_{1}$ and $G_{2}$ defined by

$$
\begin{aligned}
\left(\mathrm{G}_{1}\right)_{i} & =g^{\prime}\left(x_{n}\right) & & \text { if } c_{i}^{*}=0, \\
& =\left(1 / h c_{i}^{*}\right)\left\{g\left(x_{n}+h c_{i}^{*}\right)-g\left(x_{n}\right)\right\} & & \text { otherwise, }
\end{aligned}
$$

and 


$$
\begin{aligned}
\left(\mathbf{G}_{2}\right)_{i} & =0 & & \text { if } c_{i}^{*}=0, \\
& =g^{\prime}\left(x_{n}+h c_{i}^{*}\right) & & \text { otherwise. }
\end{aligned}
$$

Thus, the stability of the solution to Eq. (2.4) is governed by the properties of a difference equation of the form

$$
\epsilon_{n+1}=\alpha(z) \epsilon_{n}+h \beta\left(z, G_{0}, \mathbf{G}_{1}, \mathbf{G}_{2}\right)
$$

with

$$
\alpha(z)=1-\mathrm{b}^{T}(A-z I)^{-1} \mathrm{e}
$$

and

$$
\beta\left(z, G_{0}, \mathbf{G}_{1}, \mathbf{G}_{2}\right)=-G_{0}+\mathbf{b}^{T}(A-z I)^{-1} E(z)\left\{\mathbf{G}_{1}-z \mathrm{G}_{2}\right\} .
$$

To establish conditions for $S$-stability, therefore, we first prove a lemma on the stability of solutions to equations of the general form (2.8).

Lemma. Define $\epsilon\left(z, h, \epsilon_{0}\right)=\alpha(z) \epsilon_{0}+h \beta(z)$ for all complex $\epsilon_{0}$, all real $h \in(0, \bar{h}]$ and for all $z \in R$, where $R$ is the region of complex $z$ with $0<\operatorname{Re}(-z) \leqq \bar{z}$.

Then there exists a real positive $h_{0}=h_{0}\left(\bar{z}, \epsilon_{0}\right) \leqq \bar{h}$ such that

$$
\left|\epsilon\left(z, h, \epsilon_{0}\right)\right|<\left|\epsilon_{0}\right|
$$

for all $\epsilon_{0} \neq 0, h \in\left(0, h_{0}\right]$ and all $z \in R$, if and only if

(1) $|\alpha(z)|<1$ in $R$, and

(2) $\beta(z) /(1-|\alpha(z)|)$ is bounded in $R$.

Proof. (a) Necessity. If $\left|\alpha\left(z^{*}\right)\right| \geqq 1$ for $z^{*} \in R$, then, for any positive $h$, there exists an $\epsilon_{0} \neq 0$ such that $|\epsilon|>\left|\epsilon_{0}\right|$ at $z=z^{*}$ provided $\beta\left(z^{*}\right) \neq 0$. If $\beta\left(z^{*}\right)=0$, $|\epsilon| \geqq\left|\epsilon_{0}\right|$ at $z^{*}$ for all $\epsilon_{0} \neq 0$.

If $\beta(z) /(1-|\alpha(z)|)$ is not bounded in $R$, there exists $z \in R$ and $\epsilon_{0}=1-|\alpha(z)| \neq 0$ such that

$$
\frac{|\beta(z)|}{\epsilon_{0}}=\frac{|\beta(z)|}{1-|\alpha(z)|}>K
$$

for any real positive $K$. Since $|\epsilon|=\left|\epsilon_{0}\right|\left|\alpha(z)+h \beta(z) / \epsilon_{0}\right|$, for any positive $h$, there exists $K$ such that $|\epsilon|>\left|\epsilon_{0}\right|$.

(b) Sufficiency. If $\beta(z) /(1-|\alpha(z)|)$ is bounded, there exists a real positive $K$ such that

$$
\frac{|\beta(z)|}{1-|\alpha(z)|}<K
$$

for all $z \in R$. Now

$$
|\epsilon| \leqq|\alpha(z)|\left|\epsilon_{0}\right|+h|\beta(z)|=\left|\epsilon_{0}\right|-\{1-|\alpha(z)|\}\left\{\left|\epsilon_{0}\right|-\frac{h|\beta(z)|}{1-|\alpha(z)|}\right\},
$$

and, if $|\alpha(z)|<1$ in $R$, then $|\epsilon|<\left|\epsilon_{0}\right|$ for all $z \in R$, all $\epsilon_{0} \neq 0$, and all $h \in\left(0, h_{0}\right]$ where $h_{0}=\operatorname{Min}\left\{\bar{h},\left|\epsilon_{0}\right| / K\right\}$.

For a given function $g(x)$, this lemma establishes conditions for the stability of solutions to Eq. (2.8). By applying the lemma, firstly to functions $g^{\prime}(x)=0$ (for 
which $\beta \equiv 0)$, and secondly to $g(x)$ with bounded $g^{\prime}(x)$, the necessary and sufficient conditions for $A$-stability and $S$-stability may be defined.

COROLlaRY 2.1. A well-defined one-step method is A-stable if and only if $|\alpha(z)|<1$ for all $z$ with $\operatorname{Re}(z)<0$.

COROLlary 2.2. A well-defined one-step method is S-stable if and only if it is A-stable and $\beta\left(z, G_{0}, \mathbf{G}_{1}, \mathbf{G}_{2}\right) /(1-|\alpha(z)|)$ is bounded for all $z$ with $0<\operatorname{Re}(-z) \leqq \bar{z}$ (any $\bar{z}>0$ ) and for all $g(x)$ with $g^{\prime}(x)$ defined and bounded in $\left[x_{n}, x_{n}+h\right]$.

We are now in a position to state theorems which relate the conditions for $S$ stability to the values of the parameters defining a one-step method. We define the following limits as $|z| \rightarrow 0$ and $\operatorname{Re}(z)<0$ noting that the value of these limits may depend on the way in which the limit is taken:

$$
\begin{aligned}
\alpha_{0} & =\operatorname{Lt} \alpha(z)=1-\operatorname{Lt~}^{T}(A-z I)^{-1} \mathbf{e}, \\
\alpha_{1} & =\operatorname{Lt} z^{-1}\{1-|\alpha(z)|\} \\
\mathbf{b}_{0}^{* T} & =\operatorname{Lt~} \mathbf{b}^{T}(A-z I)^{-1} E(z) .
\end{aligned}
$$

In addition, it is convenient to identify the particular subset of one-step methods for which

$$
\underset{|z| \rightarrow 0}{\operatorname{Lt}} \beta\left(z, G_{0}, \mathbf{G}_{1}, \mathbf{G}_{2}\right)=0 .
$$

Since $h \beta\left(z, G_{0}, \mathbf{G}_{1}, \mathbf{G}_{2}\right)$ represents the local truncation error, methods satisfying condition (2.9) may be termed stiffly accurate. It is readily shown that for a method to be stiffly accurate it must include an abscissa $c_{r}^{*}=1$ and also

$$
\mathrm{b}_{0}^{* T}=(0,0, \cdots, 0,1)=\mathrm{e}_{r *}^{T} .
$$

It follows from the definition of $\mathrm{b}_{0}{ }^{*}$ that, if a method has a single abscissa $c_{r}=1$ and a nonsingular array $A$, the conditions for it to be stiffly accurate are

$$
c_{r}=1, \quad b_{i}=a_{r i} \quad(i=1, \cdots, r)
$$

in which case $k_{r}=h f\left(x_{n+1}, y_{n+1}\right)$.

THEOREM 2.1. A well-defined A-stable one-step method is S-stable if and only if

(a) $\left|\alpha_{0}\right|<1$ and $\mathrm{b}_{0} *$ is finite; or

(b) $\left|\alpha_{0}\right|=1, \alpha_{1} \neq 0$, and the method is stiffly accurate.

THEOREM 2.2. A well-defined S-stable one-step method is strongly $S$-stable if and only if it is strongly $A$-stable $\left(\alpha_{0}=0\right)$ and stiffly accurate.

Proofs. Let $R$ be the region of complex $z$ with $0<\operatorname{Re}(-z) \leqq \bar{z}$.

(1a) If the method is $A$-stable and $\left|\alpha_{0}\right|<1,(1-|\alpha(z)|)^{-1}$ is bounded in $R$. $\beta\left(z, G_{0}, \mathbf{G}_{1}, \mathbf{G}_{2}\right)$ is finite for all $z \in R$ and for all bounded $g^{\prime}(x)$, and is bounded if and only if $\mathrm{b}_{0}{ }^{*}$ is finite. Hence, by Corollary 2.2 , the method is $S$-stable if and only if $b_{0} *$ is finite.

(1b) If the method is $A$-stable, $\left|\alpha_{0}\right|=1$ and $\alpha_{1} \neq 0 ; z(1-|\alpha(z)|)^{-1}$ is bounded in $R$. Since $z^{-1} \beta\left(z, G_{0}, \mathbf{G}_{1}, \mathbf{G}_{2}\right)$ is bounded in $R$ for all bounded $g^{\prime}(x)$ if and only if $\mathbf{L t}_{|z| \rightarrow 0} \beta\left(z, G_{0}, \mathbf{G}_{1}, \mathbf{G}_{2}\right)=0$, by Corollary 2.2 the method is $S$-stable if and only if it is stiffly accurate.

If $\left|\alpha_{0}\right|=1$ and $\alpha_{1}=0$, it is necessary for $z^{-2} \beta\left(z, G_{0}, \mathbf{G}_{1}, \mathbf{G}_{2}\right)$ to be bounded in $R$. This requires that $\mathrm{b}_{0} *^{T} \mathrm{G}_{1}=G_{0}$ and $\mathrm{b}_{0} * *^{T} \mathrm{G}_{1}=\mathrm{b}_{0} *^{T} \mathrm{G}_{2}$, where $\mathrm{b}_{0} * * T=$ $\mathrm{Lt}_{|z| \rightarrow 0} z^{-1}\left\{\mathrm{~b}^{T}(A-z I)^{-1} E(z)-\mathrm{b}_{0} *^{T}\right\}$. If $\mathrm{b}_{0} *^{T} \mathrm{G}_{1}=G_{0}$ for all bounded $g^{\prime}(x), \mathrm{b}_{0} *=\mathrm{e}_{r^{*}}$, 
so that we require $\mathbf{b}_{0}{ }^{* * T} \mathbf{G}_{1}=\mathbf{e}_{r^{*}}{ }^{T} \mathrm{G}_{1}=g^{\prime}\left(x_{n}+h\right)$. Since $g^{\prime}\left(x_{n}+h\right)$ is not an element of $\mathbf{G}_{1}$ for positive $h$, this identity cannot hold for all bounded $g^{\prime}(x)$.

(2) From Eq. (2.8), $\left|\epsilon_{n+1}\right| \rightarrow 0$ as $|z| \rightarrow 0$ in $R$ if and only if $\alpha_{0}=0$ and $\mathrm{Lt}_{|z| \rightarrow 0} \beta\left(z, G_{0}, \mathbf{G}_{1}, \mathbf{G}_{2}\right)=0$.

3. The Stiff Order of One-Step Methods. Having established conditions for the stability of numerical solutions to equations of the form (2.4), we consider now the accuracy of such approximations. As $\operatorname{Re}(-\lambda) \rightarrow \infty$, the true solution to Eq. (2.4) tends to $g(x)$ for $x>0$, regardless of the initial condition at $x=0$. Thus, if Eq. (2.4) is stiff, a measure of the accuracy of a one-step method is provided by the difference

$$
l_{n}=y_{n+1}-g\left(x_{n+1}\right),
$$

where $y_{n+1}$ is the solution at $x_{n+1}=x_{n}+h$ to the initial-value problem

$$
y^{\prime}=g^{\prime}(x)+\lambda\{y-g(x)\}, \quad y=g\left(x_{n}\right) \text { at } x=x_{n} .
$$

The local truncation error $l_{n}$ is dependent both on $h$ and $\lambda$, as well as $g(x)$, and the asymptotic dependence

$$
l_{n} \propto h^{s+1} \lambda^{t} \text { as } \operatorname{Re}(-h \lambda) \rightarrow \infty \text { and } h \rightarrow 0
$$

defines the stiff order $(s, t)$ of a one-step method. In this section, we derive some general results on the stiff order properties of one-step methods.

The local truncation error $l_{n}$ is obtained from Eq. (2.7) by setting $\epsilon_{n}=0$, so that

$$
\begin{aligned}
l_{n} & =-h G_{0}+h \mathbf{b}^{T}(A-z I)^{-1} E(z)\left\{\mathbf{G}_{1}-z \mathbf{G}_{2}\right\} \\
& =h \beta\left(z, G_{0}, \mathbf{G}_{1}, \mathbf{G}_{2}\right) .
\end{aligned}
$$

The term $\mathrm{b}^{T}(A-z I)^{-1} E(z)$ may be expanded as a power series in $z$, giving

$$
\mathbf{b}^{T}(A-z I)^{-1} E(z)=z^{-m} \sum_{a=0}^{\infty} z^{a} \mathbf{d}_{a}^{T}
$$

where $m \geqq 0$,

$$
\begin{aligned}
\mathrm{d}_{0}^{T} & =\underset{|z| \rightarrow 0}{\mathbf{L t}} z^{m} \mathrm{~b}^{T}(A-z I)^{-1} E(z), \\
\mathrm{d}_{a}^{T} & =\underset{|z| \rightarrow 0}{\mathbf{L t}} z^{m} \mathrm{~b}^{T}(A-z I)^{-a}\left\{(A-z I)^{-1} E(z)+E(1)-E(0)\right\} .
\end{aligned}
$$

Therefore, we have

$$
h^{-1} l_{n}=-G_{0}+\mathbf{d}_{0}^{T} \mathbf{G}_{1} z^{-m}+\sum_{a=1}^{\infty}\left\{\mathbf{d}_{q}^{T} \mathbf{G}_{1}-\mathbf{d}_{a-1}^{T} \mathbf{G}_{2}\right\} z^{a-m}
$$

To establish the stiff order $(s, t)$, we consider the limiting form of Eq. (3.5):

$$
h^{-1} l_{n} \propto h^{s-t} z^{-t} \text { as } h,|z| \rightarrow 0 .
$$

In the following theorems, we assume that $g(x)$ is sufficiently differentiable and denote the $i$ th derivative at $x=x_{n}$ by $g_{n}{ }^{(i)}$. The notation $\mathrm{c}_{j}{ }^{* T}=\left(c_{1}{ }^{* i}, c_{2}{ }^{* i}, \cdots, c_{r}{ }^{* i}\right)$ is also used.

THEOREM 3.1. A stiffly accurate one-step method has stiff order $(s,-1)$ with 
$s \leqq r^{\prime}-1$, where $r^{\prime}\left(\leqq r^{*}\right)$ is the number of nonzero elements of $\mathrm{d}_{1}$.

Proof. For stiffly accurate methods, $\mathrm{Lt}_{|z| \rightarrow 0} \beta\left(z, G_{0}, \mathrm{G}_{1}, \mathrm{G}_{2}\right)=0$. Thus $h^{-1} l_{n} \rightarrow$ $z\left\{d_{1}{ }^{T} G_{1}-d_{0}{ }^{T} G_{2}\right\}$ as $|z| \rightarrow 0$, and since $d_{0} \equiv b_{0}{ }^{*}=e_{r^{*}}$ then $d_{0}{ }^{T} G_{2}=g^{\prime}\left(x_{n+1}\right)$. Expanding $g^{\prime}\left(x_{n+1}\right)$ and $\mathrm{G}_{1}$ about $x_{n}$ gives

$$
\begin{aligned}
h^{-1} l_{n} \rightarrow h^{\alpha^{\prime}} z \frac{1}{\left(q^{\prime}+1\right) !} g_{n}^{\left(a^{\prime}+1\right)}\left\{\mathrm{d}_{1}^{T} \mathrm{c}_{q^{\prime}}^{*}-\left(q^{\prime}+1\right)\right\} \quad \text { as } \quad|z|, h \rightarrow 0, \\
\text { where } \mathrm{d}_{1}^{T} \mathrm{c}_{a-1}^{*}=q \text { for } q=1,2, \cdots, q^{\prime}, \\
\neq q \text { for } q=q^{\prime}+1 .
\end{aligned}
$$

Since $q^{\prime} \leqq r^{\prime}$, then $s=q^{\prime}-1 \leqq r^{\prime}-1$ and $t=-1$.

THEOREM 3.2. A one-step method which is not stiffly accurate has stiff order $(s, m)$ with $s \leqq r^{\prime}$ for $m=0$ and $s \leqq m+r^{\prime}-1$ for $m>0$, where $r^{\prime}\left(\leqq r^{*}\right)$ is the number of nonzero elements of $\mathrm{d}_{0}$.

Proof. For $m=0$,

$$
\begin{aligned}
h^{-1} l_{n} \rightarrow \mathrm{d}_{0}^{T} \mathrm{G}_{1}-G_{0} & \text { as }|z| \rightarrow 0, \\
\rightarrow h^{\alpha^{\prime}} \frac{1}{\left(q^{\prime}+1\right) !} g_{n}^{\left(Q^{\prime}+1\right)}\left\{\mathrm{d}_{0}^{T} \mathrm{c}_{q^{\prime}}^{*}-1\right\} & \text { as } h \rightarrow 0,
\end{aligned}
$$

where

$$
\begin{aligned}
\mathrm{d}_{0}^{T} \mathrm{c}_{a-1}^{*} & =1 \text { for } q=1,2, \cdots, q^{\prime}, \\
& \neq 1 \quad \text { for } q=q^{\prime}+1 .
\end{aligned}
$$

Since $q^{\prime} \leqq r^{\prime}, s=q^{\prime} \leqq r^{\prime}$ and $t=m=0$. For $m>0$,

$$
h^{-1} l_{n} \rightarrow z^{-m} \mathrm{~d}_{0}^{T} \mathrm{G}_{1} \rightarrow h^{a^{\prime}} z^{-m} \frac{1}{\left(q^{\prime}+1\right) !} g_{n}^{\left(a^{\prime}+1\right)} \mathrm{d}_{0}^{T} \mathrm{c}_{a^{\prime}}^{*}
$$

where

$$
\begin{aligned}
\mathrm{d}_{0}^{T} \mathrm{c}_{q-1}^{*} & =0 \text { for } q=1,2, \cdots, q^{\prime}, \\
& \neq 0 \text { for } q=q^{\prime}+1 .
\end{aligned}
$$

In this case, $q^{\prime} \leqq r^{\prime}-1$, so that $s=m+q^{\prime} \leqq m+r^{\prime}-1$ and $t=m$. Since $m=0$ for $S$-stable methods, it follows that

COROLlARY 3.1. S-stable methods that are not stiffly accurate have stiff order $(s, 0)$ with $s \leqq r^{\prime}$, where $r^{\prime}$ are the number of nonzero elements of $d_{0}$.

Also, since $\left(\mathrm{d}_{0}\right)_{1}=0$ if $c_{1}{ }^{*}=0$ and $A$ is nonsingular, we have

COROLlARY 3.2. Methods that are not stiffly accurate with $c_{1}{ }^{*}=0$ and nonsingular array $A$ have stiff order $(s, m)$ with $s \leqq r^{*}-1$ for $m=0$ and $s \leqq m+r^{*}-2$ for $m>0$.

4. The S-Stability and Stiff Order of Some Classes of One-Step Method. In this section, we consider the $S$-stability and stiff order of several classes of previously published $A$-stable one-step methods based on quadrature formulae. The processes considered are the class $G$ methods of Butcher [15], based on GaussLegendre, classes IA and IIA of Ehle [8], based on Radau, and, finally, classes IIIA 
TABLE 1

Summary of the stability, stiff accuracy and orders of some classes of one-step methods

\begin{tabular}{|c|c|c|c|c|c|c|c|c|}
\hline \multirow{2}{*}{$\begin{array}{c}\text { Quadrature } \\
\text { Class }\end{array}$} & \multicolumn{4}{|c|}{ Stability } & \multirow{2}{*}{$\begin{array}{c}\text { Stiffly } \\
\text { Accurate }\end{array}$} & \multirow{2}{*}{$\begin{array}{l}\text { Order } \\
p\end{array}$} & \multicolumn{2}{|c|}{ Stiff Order } \\
\hline & $A$ & Strong- $A$ & $S$ & Strong-S & & & $s$ & $t$ \\
\hline Gauss & $\sqrt{ }$ & & & & & $2 r$ & $r$ & 0 \\
\hline Radau IA & $\sqrt{ }$ & $\sqrt{ }$ & $\sqrt{ }$ & & & $2 r-1$ & $r-1$ & 0 \\
\hline Radau IIA & $\sqrt{ }$ & $\sqrt{ }$ & $\sqrt{ }$ & $\sqrt{ }$ & $\sqrt{ }$ & $2 r-1$ & $r-1$ & -1 \\
\hline Lobatto IIIA & $\sqrt{ }$ & & $\dagger$ & & $\sqrt{ }$ & $2 r-2 *$ & $r-1^{*}$ & -1 \\
\hline Lobatto IIIB & $\sqrt{ }$ & & & & & $2 r-2$ & $r-1$ & +1 \\
\hline Lobatto IIIC & $\sqrt{ }$ & $\sqrt{ }$ & $\sqrt{ }$ & $\sqrt{ }$ & $\sqrt{ }$ & $2 r-2$ & $r-2$ & -1 \\
\hline
\end{tabular}

The absence of an entry indicates that the property does not hold.

* The methods of class IIIA have $k_{1}=h f\left(x_{n}, y_{n}\right)$ and $k_{r}=h f\left(x_{n+1}, y_{n+1}\right)$, so that, except for $n=0$, each step only requires $r-1$ evaluations of $f(x, y)$.

${ }^{\dagger}$ This class is $S(\alpha)$-stable for $\alpha \in(0, \pi / 2)$.

and IIIB of Ehle [8] and class IIIC of Chipman [11] based on the Lobatto quadrature formula. The $A$-stability of these classes has been determined by Ehle [7] and Chipman [12] inter alia, and the $S$-stability and stiff order properties are summarized in Table 1 with more detailed considerations presented in the form of a collection of theorems in this section.

Before considering the theorems in detail, we present some notation and also two lemmas which will assist in the proof of some of these theorems. We define

$$
V_{i}=\left(\begin{array}{cccc}
1 & c_{1} & \cdots & c_{1}^{i-1} \\
\vdots & \vdots & & \vdots \\
1 & \vdots & & \vdots \\
1 & c_{r} & \cdots & c_{r}^{i-1}
\end{array}\right), \quad C_{i}=\left(\begin{array}{cccc}
c_{1} & \frac{1}{2} c_{1}^{2} & \cdots & \frac{1}{2} c_{1}^{i} \\
\vdots & & & \cdot \\
c_{r} & \frac{1}{2} c_{r}^{2} & \cdots & \frac{1}{2} c_{r}^{i}
\end{array}\right)
$$

to be $r \times i$ matrices, with the suffix $i$ denoting the number of columns,

$$
\Gamma_{r}=\left(\begin{array}{cccc}
1 & & & \\
& \frac{1}{2} & & \\
& & \ddots & \\
& & & \frac{1}{r}
\end{array}\right), \quad \Gamma_{r}^{*}=\left(\begin{array}{llll}
1 & & & \\
& 2 & & \\
& & \ddots & \\
& & & r
\end{array}\right)
$$

to be $r \times r$ diagonal matrices, and

$$
\boldsymbol{\gamma}_{i}^{T}=\left[1, \frac{1}{2}, \cdots, 1 / i\right], \quad \boldsymbol{\gamma}_{i}^{* T}=[1,2, \cdots, i]
$$

to be $i$ vectors. We take $\mathrm{e}$ to be the unit vector and $\mathrm{e}_{i}$ to be the vector having the $i$ th element unity with the remaining elements zero: the length of both these vectors is assumed to be that applicable at the time of use. We also use Butcher's notation [15] for representing certain conditions satisfied by the parameters $a_{i j}$ and $b_{i}$. Finally, 
we omit the asterisk from the abscissa $c_{i}$ and also from the number of stages of the methods, since all classes under consideration here have distinct abscissae.

Lemma 4.1. An A-stable one-step method is S-stable if $A$ is positive definite and $\alpha_{0}<1$.

Lemma 4.2. A strongly A-stable one-step method is strongly S-stable and stiffy accurate if

(1) $A$ is positive definite,

(2) $\mathbf{b}^{T} A^{-1}=\mathbf{e}_{r}{ }^{T}$,

(3) the method contains a single abscissa $c_{r}=1$.

Proof.

$$
\begin{aligned}
\mathbf{b}_{0}^{* T} & =\underset{|z| \rightarrow 0}{\operatorname{Lt}} \mathbf{b}^{T}(A-z I)^{-1} E(z) \\
& =\mathbf{b}^{T} A^{-1} E(0)=\mathrm{e}_{r}^{T} E(0)=\mathrm{e}_{r}^{T} .
\end{aligned}
$$

Consequently, the method is stiffly accurate and hence, by Theorem 2.2 , strongly $S$-stable.

THEOREM 4.1. The processes of class $\mathrm{G}$ are not $S$-stable and have stiff order $(r, 0)$. Proof. For this class, $\left|\alpha_{0}\right|=1$, since $\alpha(1 / z)$ reduces to the diagonal Padé approximation to $\exp (1 / z)$. As this class does not have an abscissa of unity, it cannot be stiffly accurate, so, by Theorem 2.1, the processes are not $S$-stable. Now

$$
\begin{aligned}
\mathbf{d}_{0}^{T} & =\underset{|z| \rightarrow 0}{\mathbf{L t}} \mathbf{b}^{T}(A-z I)^{-1} E(z)=\mathbf{b}^{T} A^{-1} E(0) \\
& =\gamma_{r}^{T}\left(A V_{r}\right)^{-1} E(0) \quad \text { by } B(r) \\
& =\gamma_{r}^{T} C_{r}^{-1} E(0) \quad \text { by } C(r) \\
& =\gamma_{r}^{T} \Gamma_{r}^{-1} V_{r}^{-1}=\mathrm{e}^{T} V_{r}^{-1} \Rightarrow t \leqq 0 .
\end{aligned}
$$

Since this class does not contain an abscissa of unity, we have $t=0$. As $\mathrm{d}_{0}{ }^{T} V_{\mathrm{r}}=\mathrm{e}^{T}$, by Theorem 3.2, $s=r$.

THEOREM 4.2. The processes of class IA are S-stable and have stiff order $(r-1,0)$.

Proof. The $S$-stability of this class is a direct consequence of Lemma 4.1. Since this class does not have an abscissa of unity, it cannot be stiffly accurate and so, by Corollary 3.2, the stiff order is $(s, 0)$ where $s \leqq r-1$. Now

$$
\begin{aligned}
& \mathrm{d}_{0}^{T} V_{r-1}=\mathbf{b}^{T} A^{-1} E(0) V_{r-1} \\
& =\mathbf{b}^{T} A^{-1} C_{r-1} \Gamma_{r-1}^{*} \\
& =\mathbf{b}^{T} V_{r-1} \Gamma_{r-1}^{*} \text { by } C(r-1) \\
& =\gamma_{r-1}^{T} \Gamma_{r-1}^{*} \quad \text { by } B(r-1) \\
& =\mathrm{e}^{T} \text {. }
\end{aligned}
$$

Hence, by Theorem 3.2, $s=r-1$.

THEOREM 4.3. The processes of class IIA are strongly S-stable, stiffly accurate and have stiff order $(r-1,-1)$.

Proof. By $B(r)$,

$$
\begin{aligned}
\mathbf{b}^{T} & =\gamma_{r}^{T} V_{r}^{-1}=\mathrm{e}_{r}^{T} C_{r} V_{r}^{-1} \\
& =\mathbf{e}_{r}^{T} A \text { by } C(r) .
\end{aligned}
$$


Hence, by Lemma 4.2 , the class is strongly $S$-stable and stiffly accurate which implies it has stiff order $(s,-1)$ with $s \leqq r-1$. As $E(0) V_{r}=C_{r} \Gamma_{r}{ }^{*}$,

$$
\begin{aligned}
\mathrm{d}_{1}^{T} V_{r}=\mathrm{b}^{T} A^{-2} E(0) V_{r} & =\mathrm{e}_{r}^{T} A^{-1} C_{r} \Gamma_{r}^{*} \\
& =\mathrm{e}_{r}^{T} V_{r} \Gamma_{r}^{*} \text { by } C(r) \\
& =\mathrm{e}^{T} \Gamma_{r}^{*} \\
& =\gamma_{r}^{* T} .
\end{aligned}
$$

Hence, by Theorem 3.1, $s=r-1$.

THEOREM 4.4. The processes of class IIIA are stiffly accurate, have stiff order $(r-1,-1)$, and are not $S$-stable but are $S(\alpha)$-stable for $\alpha \in(0, \pi / 2)$.

Proof. For this class, $\left|\alpha_{0}\right|=1$ and $\alpha_{1}=0$ in the $\operatorname{limit} \operatorname{Im}(\lambda) \rightarrow \infty$ (diagonal Padé approximation [12]), and hence, from Theorem 2.1, this class is not $S$-stable. However, for $\alpha \in(0, \pi / 2), \alpha_{1} \neq 0$ and hence by Theorem 2.1 this class is $S(\alpha)$-stable. Now

$$
\begin{aligned}
\mathrm{b}_{0}^{* T} & =\underset{|z| \rightarrow 0}{\operatorname{Lt}} \mathbf{b}^{T}(A-z I)^{-1} E(z) \\
& =\underset{|z| \rightarrow 0}{\operatorname{Lt}} \gamma_{r}^{T} V_{r}^{-1}(A-z I)^{-1} E(z) \quad \text { by } B(r) \\
& =\underset{|z| \rightarrow 0}{\operatorname{Lt}} \gamma_{r}^{T}\left(C-z V_{r}\right)^{-1} E(z) \quad \text { by } C(r) \\
& =\gamma_{r}^{T} \Omega^{-1} \quad \text { where } \quad \Omega_{i j}=c_{i}^{i-1} / j \quad(i, j=1,2, \cdots, r) .
\end{aligned}
$$

As $\gamma_{r}{ }^{T}=\mathrm{e}_{r}{ }^{T} \Omega$, it follows that $\mathrm{b}_{0}{ }^{*}=\mathrm{e}_{r}$, so that this class is stiffly accurate. As it is stiffly accurate, by Theorem 3.1 , it has stiff order $(s,-1)$ with $s \leqq r-1$.

Let $A^{*}$ be the principal minor of $A$ and $\mathrm{a}^{*^{T}}=\left(a_{21}, \cdots, a_{r 1}\right)$, so that

$$
A=\left(\begin{array}{cc}
0 & 0 \\
\mathrm{a}^{*} & A^{*}
\end{array}\right)
$$

and let $C^{*}$ and $V^{*}$ denote the corresponding nonsingular minors of $C_{r}$ and $V_{r}$ respectively. Since, by $C(r), A=C_{r} V_{r}^{-1}$, it follows that $A^{*}=C^{*} V^{*-1}$. Now

$$
(A-z I)^{-1}=\left(\begin{array}{cc}
-z^{-1} & 0 \\
z^{-1}\left(A^{*}-z I\right)^{-1} \mathrm{a}^{*} & \left(A^{*}-z I\right)^{-1}
\end{array}\right)
$$

and

$$
(A-z I)^{-1} E(z)+E(1)-E(0)=\left(\begin{array}{cc}
0 & 0 \\
-\left(A^{*}-z I\right)^{-1} \mathrm{a}^{*} & \left(A^{*}-z I\right)^{-1} D(c)
\end{array}\right)
$$

where $D(c)$ is a diagonal matrix with elements $c_{i}(i=2, \cdots, r)$. By $B(r), \mathrm{b}^{T}=\gamma_{r}{ }^{T} V_{r}{ }^{-1}$ $=\mathrm{e}_{r}{ }^{T} C_{r} V_{r}^{-1}=\mathrm{e}_{r}{ }^{T} A$, so that

$$
\mathrm{b}^{T}-z \mathrm{e}_{r}^{T}=\mathrm{e}_{r}^{T}(A-z I)
$$

and

$$
\mathrm{b}^{T}(A-z I)^{-1}=\mathrm{e}_{r}^{T}+z \mathrm{e}_{r}^{T}(A-z I)^{-1}=\mathrm{e}_{r}^{T}+\left[\mathrm{e}_{r-1}^{T} A^{*-1} \mathrm{a}^{*}, 0, \cdots, 0\right]+O(z) .
$$


Hence

$$
\begin{aligned}
\mathbf{d}_{1}^{T} & =\underset{|z| \rightarrow 0}{\operatorname{Lt}} \mathbf{b}^{T}(A-z I)^{-1}\left\{(A-z I)^{-1} E(z)+E(1)-E(0)\right\} \\
& =\left[-\mathrm{e}_{r-1}^{T} A^{*^{-1}} \mathrm{a}^{*}, \mathrm{e}_{r-1}^{T} A^{*^{-1}} D(c)\right] \\
& =\left[1-\mathrm{e}_{r-1}^{T} A^{*^{-1}} D(c) \mathrm{e}, \mathrm{e}_{r-1}^{T} A^{*^{-1}} D(c)\right]
\end{aligned}
$$

since $\mathrm{a}^{*}=\left\{D(c)-A^{*}\right\}$ e. Thus, since $A^{*-1} D(c) V^{*}=A^{*^{-1}} C^{*} D(i)=V^{*} D(i)$ where $D(i)$ is the diagonal matrix with elements $2, \cdots, r$,

$$
\mathrm{d}_{1}^{T} V_{r}=\left[1, \mathrm{e}_{r-1}^{T} V^{*} D(i)\right]=\gamma_{r}^{* T} \text {. }
$$

Hence, by Theorem 3.1, $s=r-1$.

THEOREM 4.5. The processes of class IIIB are not S-stable and have stiff order $(r-1,1)$.

Proof. Let

$$
A=\left(\begin{array}{ll}
A^{*} & 0 \\
\mathrm{a}^{* T} & 0
\end{array}\right)
$$

where $A^{*}$ may be shown to be a nonsingular minor of $A$. Then

$$
(A-z I)^{-1}=\left|\begin{array}{cc}
\left(A^{*}-z I\right)^{-1} & 0 \\
z^{-1} \mathrm{a}^{* T}\left(A^{*}-z I\right)^{-1} & -z^{-1}
\end{array}\right| .
$$

Let $\mathrm{b}^{*}$ denote the first $(r-1)$ elements of $\mathrm{b}$, and \{\}$_{i}$ denote the $i$ th component of the vector. Then

$$
\begin{aligned}
\mathbf{d}_{0}^{T} & =\underset{|z| \rightarrow 0}{\mathbf{L t}} z \mathbf{b}^{T}(A-z I)^{-1} E(z) \\
& =\underset{|z| \rightarrow 0}{\mathbf{L t}}\left[-z\left\{\Delta^{T}(z)\right\}_{1},\left\{\Delta^{T}(z) D(c)\right\}_{i},-b_{r}\right] \quad(j=2, \cdots, r-1),
\end{aligned}
$$

where $\Delta^{T}(z)=\left(z \mathrm{~b}^{*}+b_{r} \mathrm{a}^{*}\right)^{T}\left(A^{*}-z I\right)^{-1}$ and $D(c)$ is a diagonal matrix with elements $c_{i}(i=1, \cdots, r-1)$, and so

$$
\mathbf{d}_{0}^{T}=\left[0, b_{r}\left\{\mathbf{a}^{*^{T}} A^{*^{-1}} D(c)\right\}_{i},-b_{r}\right] \quad(j=2, \cdots, r-1) .
$$

Since, by $B(r), b_{r} \neq 0, \mathrm{~d}_{0}{ }^{T} \neq 0$ so that $t=1$. It follows that $\mathrm{b}_{0}{ }^{*}$ is not finite and the class is not $S$-stable.

By $C(r-2), A V_{r-2}=C_{r-2}$ so that

$$
A^{*} V_{r-2}^{*}=C_{r-2}^{*} \quad \text { and } \quad a^{*^{T}} V_{r-2}^{*}=\gamma_{r-2}^{T},
$$

where

$$
V_{r-2}=\left(\begin{array}{c}
V_{r-2}^{*} \\
\mathrm{e}^{T}
\end{array}\right) \quad \text { and } \quad C_{r-2}=\left(\begin{array}{c}
C_{r-2}^{*} \\
\gamma_{r-2}^{T}
\end{array}\right) .
$$

Thus, since $D(c) V_{r-2} *=C_{r-2} * \Gamma_{r-2} *$,

$$
\begin{aligned}
\mathrm{d}_{0}^{T} V_{r-2} & =b_{r}\left[\mathrm{a}^{*^{T}} A^{*^{-1}} D(c),-1\right] V_{r-2} \\
& =b_{r}\left[\mathrm{a}^{*^{T}} A^{*^{-1}} C_{r-2}^{*} \Gamma_{r-2}^{*}-\mathrm{e}^{T}\right]=b_{r}\left[\gamma_{r-2}^{T} \Gamma_{r-2}^{*}-\mathrm{e}^{T}\right]=0 .
\end{aligned}
$$

Hence, $s=r-1$. 
THEOREM 4.6. The processes of class IIIC are strongly S-stable, stiffy accurate and have stiff order $(r-2,-1)$.

Proof. From the derivation of the coefficients of this class, $\mathrm{b}^{T}=\mathrm{e}_{r}{ }^{T} A$, and so, by Lemma 4.2 , the class is strongly $S$-stable and stiffly accurate. Since this class is stiffly accurate, by Theorem 3.1 , it has stiff order $(s,-1)$ with $s \leqq r-1$. Now

$$
\begin{aligned}
\mathrm{d}_{1}^{T} V_{r} & =\mathrm{b}^{T} A^{-2} E(0) V_{r}=\mathrm{e}_{r}^{T} A^{-1} C_{r} \Gamma_{r}^{*} \\
& =\mathrm{e}_{r}^{T} V_{r}^{*} \Gamma_{r}^{*} \quad \text { by } C(r-1), \text { where } V_{r}^{*}=\left[\begin{array}{ll}
V_{r-1} \mathrm{v}
\end{array}\right], \\
& =\left[\mathrm{e}^{T}, v_{r}\right] \Gamma_{r}^{*} \\
& \left.=\left[\gamma_{r-1}^{* T}, 1\right] \quad \text { since } v_{r}=1 / r \text { (Chipman }[12]\right) .
\end{aligned}
$$

Thus, by Theorem 3.1, we have $s=r-2$.

5. Discussion. To conclude this paper, we consider the implications of the various stability and accuracy concepts that we have defined. At the same time, we compare the properties of the classes of one-step methods considered in the previous section, and relate these properties to the performance of the methods on a test problem of the form (2.4).

It should be borne in mind that, in discussing the behaviour of solutions to a stiff equation (2.4), we are at the same time discussing the way in which the solutions to the stiff components of nonlinear systems of differential equations tend to behave.

\section{TABLE 2}

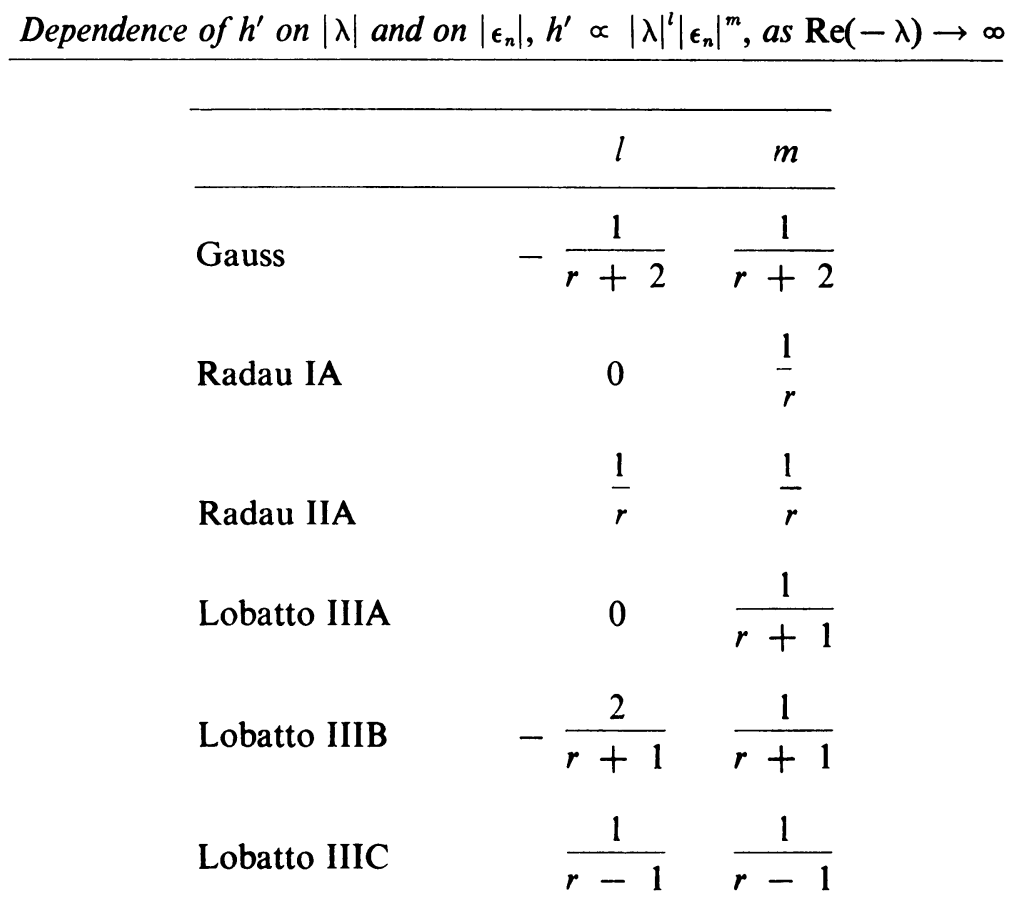




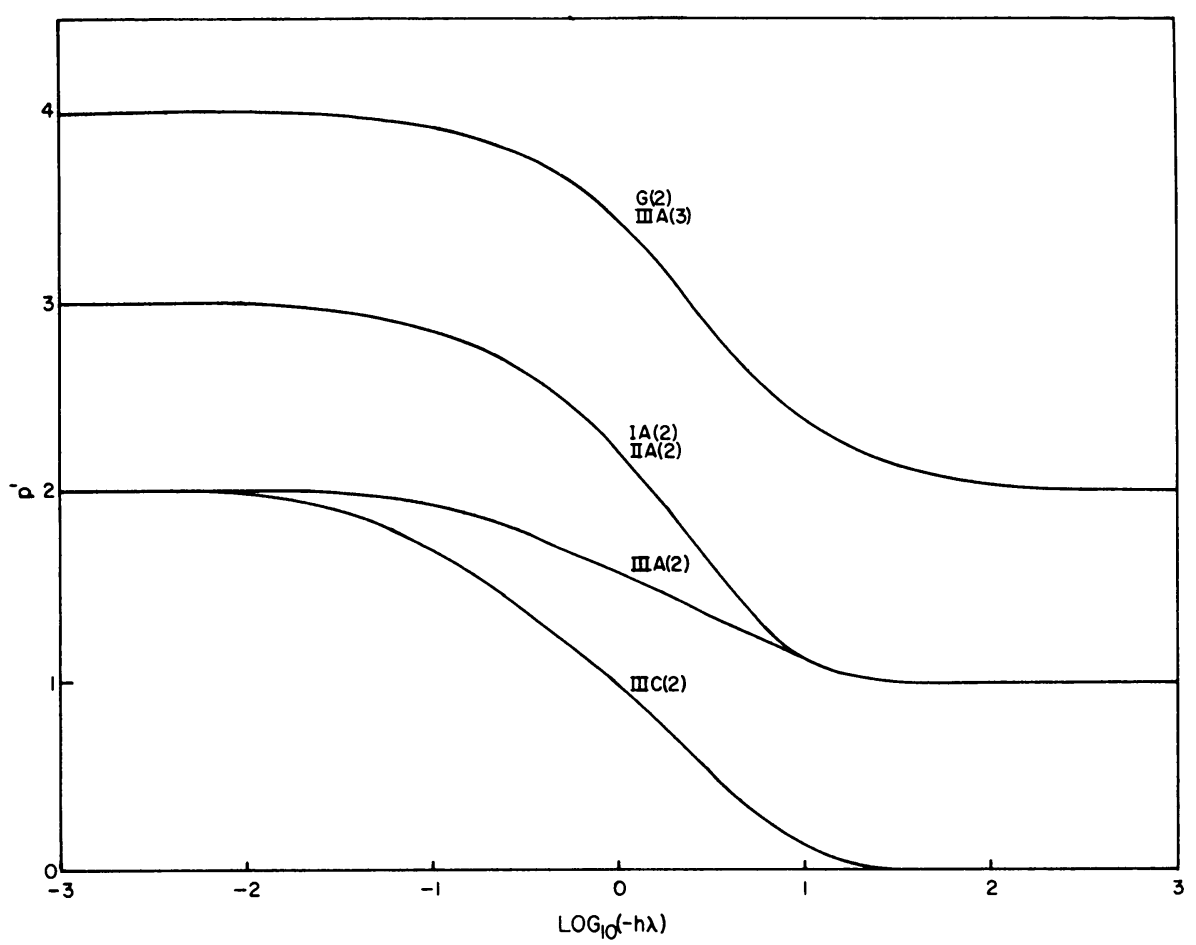

FIG. I - The apparent order $p^{\prime}=L_{h \rightarrow 0}\left\{L L_{2}\left|\frac{L_{0}(2 h)}{2 l_{0}(h)}\right|\right\}$ of various one-step methods when solving example 1. G(2) signifies Gauss 2-stage method, etc.

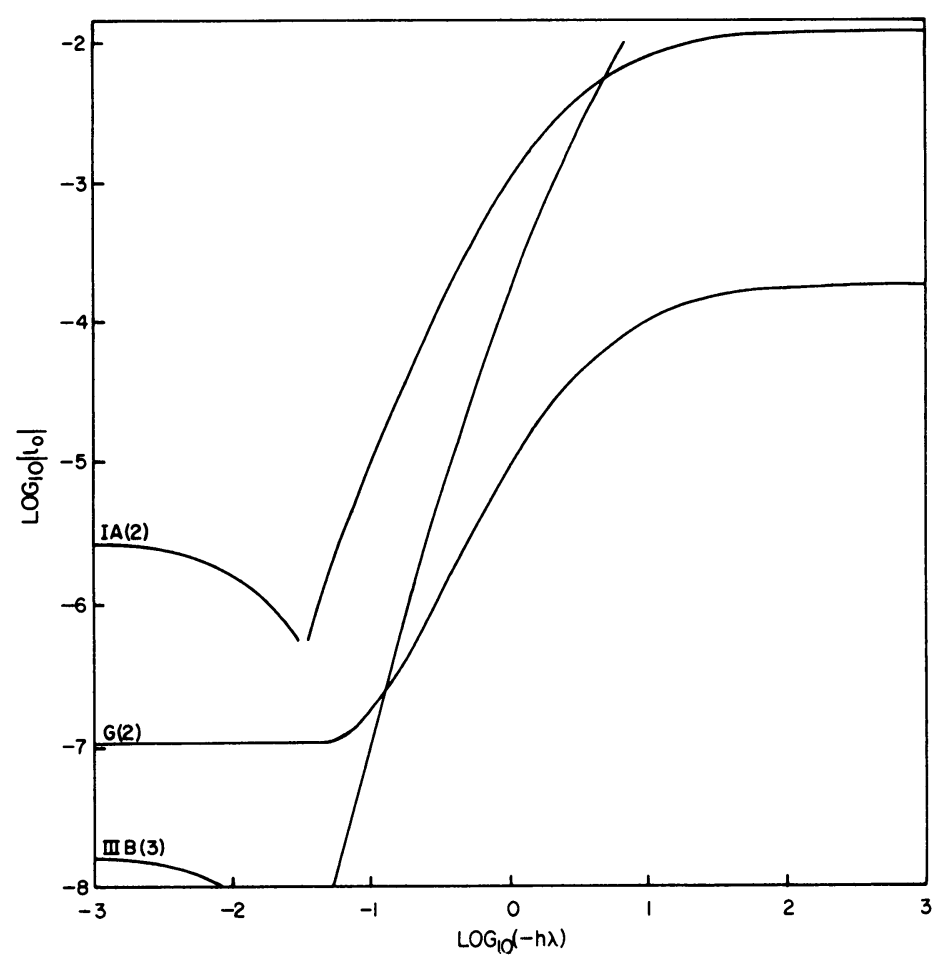

FIG.2-Local truncation errors $\mathrm{I}_{0}$ for various one-step methods that are not stiffly accurate, when solving example I with $h=0.1$ 
In Section 2, an $S$-stability property for a one-step method is defined which ensures that a range of positive step-sizes $(0, \bar{h})$ exists which gives stable solutions to Eq. (2.4) with bounded $g^{\prime}(x)$, for any $\lambda$ with $\operatorname{Re}(\lambda) \leqq-\bar{\lambda}<0$. Thus, if $h^{\prime}=$ $h^{\prime}\left(\lambda,\left|\epsilon_{n}\right|, g\right)$ denotes the upper limit to the range of stability $\left(0, h^{\prime}\right)$ for given $\lambda, S$ stability guarantees that $h^{\prime} \rightarrow 0$ as $\operatorname{Re}(-\lambda) \rightarrow \infty$, while, with strong $S$-stability, $h^{\prime} \rightarrow \infty$ as $\operatorname{Re}(-\lambda) \rightarrow \infty$.

The asymptotic dependence of $h^{\prime}$ on $\lambda$ and on $\left|\epsilon_{n}\right|$ as $\operatorname{Re}(-\lambda) \rightarrow \infty$, which is of the form $h^{\prime} \propto|\lambda|^{l}\left|\epsilon_{n}\right|^{m}$, is given for various $r$-stage one-step methods in Table 2 .

The negative values of $l$ for the Gauss and the Lobatto class IIIB methods reflects the lack of $S$-stability of these classes (Table 1 ). In order to give stable solutions to Eq. (5.1), the Lobatto IIIB methods, in particular, would require considerable reductions in the step-size as the stiffness, $\operatorname{Re}(-\lambda)$, in Eq. (5.1) increases. While the inverse $\lambda$-dependence of the Gaussian methods is fairly weak, the stability of this class on very stiff practical problems is not as good as the $S(\alpha)$-stable Lobatto IIIA class, even though the $A$-stability properties of the two classes are identical. The positive $l$-dependence of the Radau IIA and Lobatto IIIC method is a measure of the strong $S$-stability of these stiffly accurate classes.

In Section 3, the asymptotic form of the local truncation error, as $h \operatorname{Re}(-\lambda) \rightarrow \infty$, is derived as

$$
l_{n} \propto h^{s+1} \lambda^{t} g_{n}^{(s-t)} .
$$

The upper limits derived for $s$, and the $s$-values obtained for the various classes indicate that the effective order of one-step methods applied to Eq. (2.4), with $\operatorname{Re}(-h \lambda)$ large, is generally much lower than the order $p$ that can be achieved for nonstiff problems. Figure 1 shows the apparent order of various methods when solving

Example 1 (Seinfeld et al. [16]).

$$
\begin{aligned}
y^{\prime} & =g^{\prime}(x)+\lambda\{y-g(x)\}, \quad y=g(0) \quad \text { at } x=0 . \\
g(x) & =10-(10+x) e^{-x}, \quad \lambda \text { real. }
\end{aligned}
$$

For one-step methods that are not stiffly accurate, the change in the effective order results in a much lower level of accuracy for $h \operatorname{Re}(-\lambda)$ large compared with that in the nonstiff region (Figure 2). In contrast, the errors given by the stiffly accurate methods (Figure 3) tend to zero as $\operatorname{Re}(-\lambda) \rightarrow \infty$, so that the reduction in order in the stiff region is offset, to a varying extent, by the inverse dependence on $|\lambda|$. Clearly, the stiff accuracy property of one-step methods increases in significance with the degree of stiffness in Eq. (2.4).

If we use a one-step method of stiff order $(s, t)$ to solve Eq. (2.4) with $g(x)$ a polynomial of degree $(s-t)$, the error equation (2.8) is independent of $x$ and the dependence of the global error $\epsilon_{n}$ on $h$ and $|\lambda|$ as $\operatorname{Re}(-\lambda) \rightarrow \infty$ may be determined (Table 3). For strongly $A$-stable methods, the global errors tend to the local truncation errors, while for $\alpha_{0}=-1$, successive errors show a cancellation effect. For $\alpha_{0}=1$, however, $\epsilon_{n}$ increases linearly with $n$.

Comparing the properties of the various classes of one-step methods, the Lobatto IIIA methods would seem to be the most accurate of the $S$ - or $S(\alpha)$-stable stiffly accurate classes, taking into account that the $r$-stage IIIA process is equivalent, in terms of computational effort, to an $(r-1)$-stage process. In particular, IIIA methods 


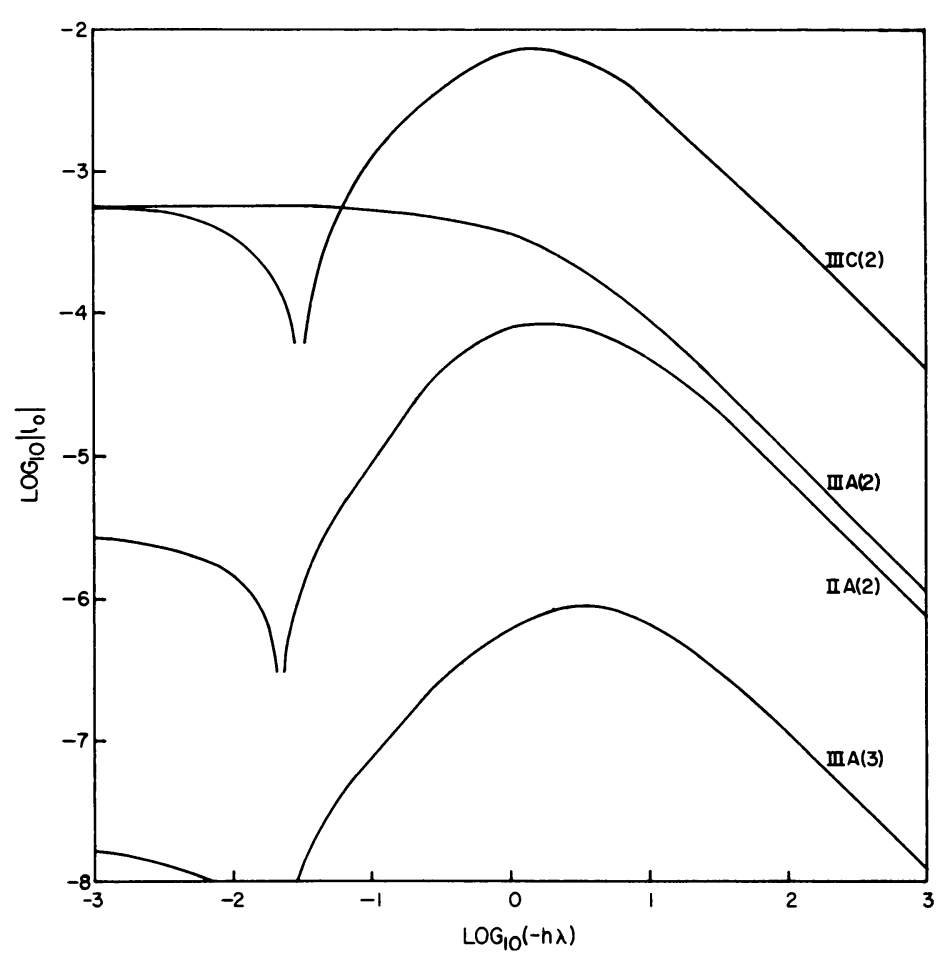

FIG.3- Local truncation errors $l_{0}$ for various stiffly accurate one-step methods, when solving example I with $h=0.1$

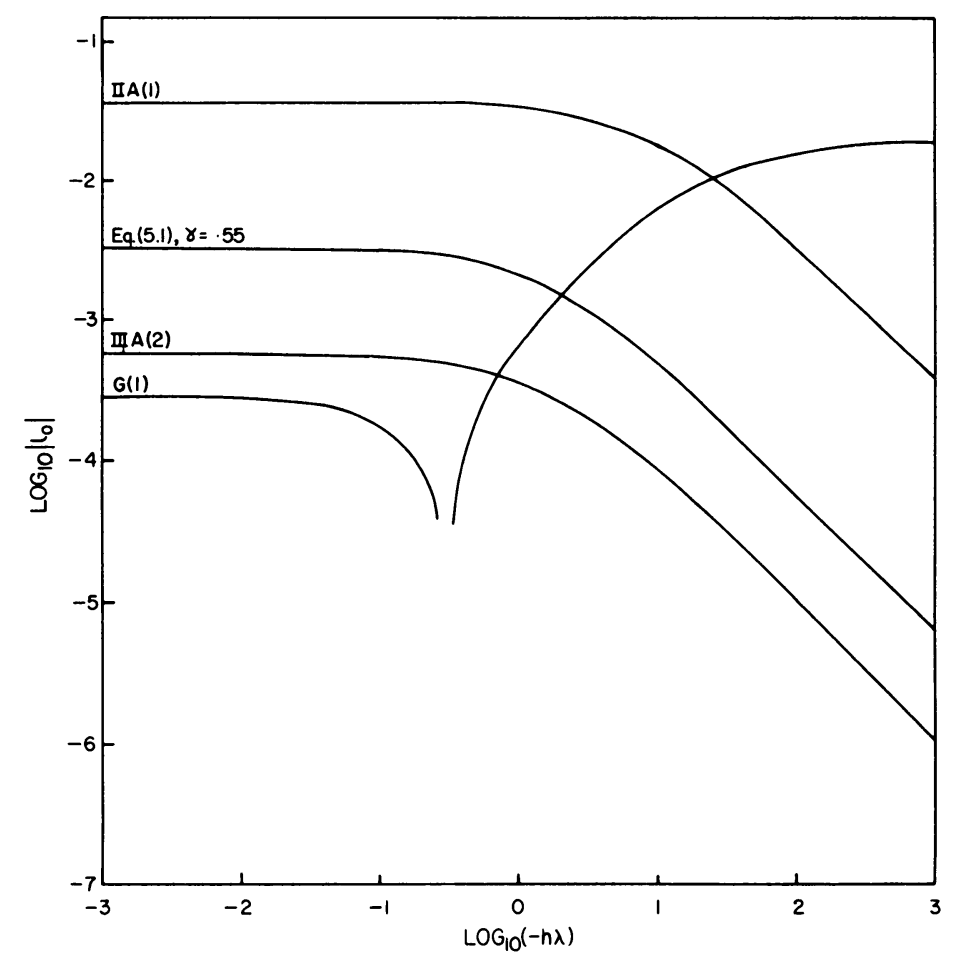

FIG.4-Local truncation error $l_{0}$ for some single-stage one-step methods, when solving example I with $h=0.1$ 
TABLE 3

Asymptotic dependence of $\epsilon_{n}$ as $\operatorname{Re}(-\lambda) \rightarrow \infty$

\begin{tabular}{lll}
\hline & \multicolumn{1}{c}{$r$ even } & \multicolumn{1}{c}{$r$ odd } \\
\hline Gauss & $n h^{r+1} g^{(r+1)}$ & $\begin{array}{l}h^{r+1} g^{(r+1)}(n \text { odd }) ; \\
n h^{r}|\lambda|^{-1} g^{(r+1)}(n \text { even })\end{array}$ \\
Radau IA & $h^{r} g^{(r)}$ & $h^{r} g^{(r)}$ \\
Radau IIA & $h^{r}|\lambda|^{-1} g^{(r+1)}$ & $h^{r}|\lambda|^{-1} g^{(r+1)}$ \\
Lobatto IIIA & $h^{r}|\lambda|^{-1} g^{(r+1)}(n$ odd $) ;$ & $n h^{r}|\lambda|^{-1} g^{(r+1)}$ \\
Lobatto IIIB & $n h^{r-1}|\lambda|^{-2} g^{(r+1)}(n$ even $)$ & \\
& $h^{r}|\lambda| g^{(r-1)}(n$ odd $) ;$ & $n h^{r}|\lambda| g^{(r-1)}$ \\
Lobatto IIIC & $n h^{r-1} g^{(r-1)}(n$ even $)$ & \\
& $h^{r-1}|\lambda|^{-1} g^{(r)}$ & $h^{r-1}|\lambda|^{-1} g^{(r)}$ \\
\hline
\end{tabular}

with an even number of stages should have a small error propagation effect and, further, for the IIIA methods there is no practical difference between $S(\alpha)$ - and $S$-stability on real problems. Note that for $r=2$ we have the trapezoidal rule.

Strongly $S$-stable methods have advantages in that the local truncation errors are very quickly damped out for $h \operatorname{Re}(-\lambda)$ large, and the methods are likely to remain stable when applied to equations of the form (1.8) having variable $\lambda(x)$. Gourlay [17] has shown that for Eq. (1.8) with $g(x)=0$ and $\operatorname{Re}(-\lambda(x))$ large but decreasing, the trapezoidal rule is only stable for a restricted range of step-sizes $h$. Similar restrictions must apply to all one-step methods, involving more than one abscissae, for which $\left|\alpha_{0}\right|=1$.

The suggested replacement of the trapezoidal rule by the implicit midpoint rule (the single-stage Gaussian process $G(1)$ ) [17] overcomes the stability problem associated with $\lambda(x)$, but since the $G(1)$ method is not stiffly accurate (or $S$-stable), there can be a considerable loss of accuracy on stiff problems (Figure 4). We would recommend replacing the Lobatto IIIA methods by other classes of methods that are stiffly accurate and either strongly $S$-stable or $S$-stable with $\left|\alpha_{0}\right|<1$.

Of the methods that are stiffly accurate and strongly $S$-stable, the Radau IIA methods should be more accurate than the Lobatto IIIC processes (Figure 3). Note that, for $r=1$, the IIA process is the backward Euler method. However the IIA processes are significantly less accurate than the IIIA processes, and a compromise between accuracy and strong $S$-stability, giving a class of stiffly accurate methods with $0<\left|\alpha_{0}\right|<1$, is worth considering.

One such family of intermediate methods may be defined by

$$
A=\left(\begin{array}{cc}
0 & 0 \\
1-\gamma & \gamma
\end{array}\right), \quad \begin{array}{ll}
\mathrm{b}^{T}=(1-\gamma, \gamma), \\
\mathrm{c}^{T}=(0,1),
\end{array}
$$

with $0.5<\gamma<1$. This first-order method is effectively single-stage, with $\alpha_{0}=$ $-(1-\gamma) / \gamma$. It may be easily shown to be $S$-stable and stiffly accurate, with generally smaller truncation error than the IIA(1) method, as shown in Figure 4 using $\gamma=$ 
0.55. The authors have in fact used this method extensively (with $\gamma=0.55$ ) to obtain stable solutions of reasonable accuracy to large nonlinear systems of stiff equations arising in the field of chemical kinetics [14].

Acknowledgments. The authors would like to express their thanks to Dr. S.P. Norsett, for pointing out that the class IIIA processes are only $S(\alpha)$-stable, and also to Miss J. Galvin, for carrying out the numerical calculations.

Shell Research Ltd.

Thornton Research Centre

Chester CHI 3SH, England

1. G. G. DAHLQUIST, "A special stability problem for linear multistep methods," Nordisk Tidskr. Informationsbehandling (BIT), v. 3, 1963, pp. 27-43. MR 30 \#15.

2. O. B. WIDLUND, "A note on unconditionally stable linear multistep methods," Nordisk Tidskr. Informationsbehandling (BIT), v. 7, 1967, pp. 65-70. MR 35 \#6373.

3. S. P. NORSETT, "A criterion for $\boldsymbol{A}(\alpha)$-stability of linear multistep methods," Nordisk Tidskr. Informationsbehandling (BIT), v. 9, 1969, pp. 259-263. MR 41 \#1227.

4. C. W. GEAR, The Automatic Integration of Stiff Ordinary Differential Equations (With Discussion), Proc. IFIP Congress Information Processing 68 (Edinburgh 1968), vol. 1; Mathematics, Software, North-Holland, Amsterdam, 1969, pp. 187-193. MR 41 \#4808.

5. C. E. TREANOR, "A method for the numerical integration of coupled first-order differential equations with greatly different time constants," Math. Comp., v. 20, 1966, pp. 39-45. MR 33 \#889.

6. S. P. NorsetT, An A-Stable Modification of the Adams-Bashforth Methods, Conf. on the Numerical Solution of Differential Equations (Dundee, Scotland, June 1969), Springer, Berlin, 1969, pp. 214-219. MR 42 \#2673.

7. B. L. EHLE, "High-order $A$-stable methods for the numerical solution of systems of differential equations," Nordisk Tidskr. Informationsbehandling (BIT), v. 8, 1968, pp. 276278. MR 39 \#1119.

8. B. L. Ehle, On Padé Approximations to the Exponential Function and A-Stable Methods for the Numerical Solution of Initial Value Problems, Report CSRR 2010, University of Waterloo, Department of Applied Analysis and Computer Science, March 1969.

9. O. AXELSSON, "A class of $A$-stable methods," Nordisk Tidskr. Informationsbehandling (BIT), v. 9, 1969, pp. 185-199. MR $40 \# 8266$.

10. O. Axelsson, "A note on a class of strongly $A$-stable methods," Nordisk Tidskr. Informationsbehandling (BIT), v. 12, 1972, pp. 1-4.

11. F. H. ChIPMAN, " $A$-stable Runge-Kutta processes," Nordisk Tidskr. Informationsbehandling (BIT), v. 11, 1971, pp. 384-388. MR 45 \#4648.

12. F. H. ChIPMAN, Numerical Solution of Initial Value Problems Using A-Stable RungeKutta Processes, Report CSRR 2042, University of Waterloo, Department of Applied Analysis and Computer Science, June 1971.

13. H. A. WatTs \& L. F. SHampine, " $A$-stable block implicit one-step methods," Nordisk Tidskr. Informationsbehandling (BIT), v. 12, 1972, pp. 252-266.

14. M. P. Halstead, A. Prothero \& C. P. Quinn, "A mathematical model of the coolflame oxidation of acetaldehyde," Proc. Roy. Soc. London Ser. A, v. 322, 1971, pp. 377-403.

15. J. C. BuTcher, "Implicit Runge-Kutta processes," Math. Comp., v. 18, 1964, pp. 50-64. MR 28 \#2641.

16. J. H. Seinfeld, L. Lapidus \& M. Hwang, "Review of numerical integration techniques for stiff ordinary differential equations," Ind. Eng. Chem. Fundamentals, v. 9, 1970, pp. 266-275.

17. A. R. Gourlay, "A note on trapezoidal methods for the solution of initial value problems," Math. Comp., v. 24, 1970, pp. 629-633. MR 43 \#1433. 